\title{
COVID-19 Cases and Deaths: A Comparison among Bangladesh, India and Pakistan
}

\author{
Shipra Banik ${ }^{1}$, B. M. Golam Kibria ${ }^{2 *}$ \\ ${ }^{1}$ Department of Physical Sciences, Independent University, Bangladesh Dhaka, Bangladesh. \\ ${ }^{2}$ Department of Mathematics and Statistics, Florida International University University Park, Miami FL. 33199, USA.
}

*Corresponding Author: B. M. Golam Kibria, Department of Mathematics and Statistics, Florida International University University Park, Miami FL. 33199, USA.

Received date: January 16, 2021 ; Accepted date: March 24, 2021; Published date: March 26,2021

Citation: S Banik, B. M. Golam Kibria. (2021) Psychiatric Stigma in Developing Societies. Biomedical Research and Clinical Reviews. 3(5); DOI: $10.31579 / 2692-9406 / 043$

Copyright: (C) 2021 B. M. Golam Kibria, This is an open-access article distributed under the terms of the Creative Commons Attribution License, which permits unrestricted use, distribution, and reproduction in any medium, provided the original author and source are credited.

\begin{abstract}
This paper compares the number of infected cases and deaths of an ongoing pandemic of COVID-19 outbreak for Bangladesh, India and Pakistan for the period of March 8, 2020 to September 21, 2020. Comparisons among countries using absolute numbers are not comparable due to different factors, such as population sizes, rates of per 100,000 and also because not all countries are affected equally and at the same time. Following Middelburg and Rosendaal (2020), we graphically compare the number of cases and deaths expressed as a percentage of the cases and deaths on the reference day 25 after the first reported death. To see the impact of reference days, several later reference days are also considered in this study. From these comparisons, clear differences were observed among countries. Among these three countries, it is observed that Bangladesh had the most extreme flattening of the curve, followed by Pakistan and then India. We observed that the epidemic developed in India much more rapidly as compare to Bangladesh and Pakistan.
\end{abstract}

Keywords: Bangladesh; Covid-19; India; Mortality; Pakistan; Pandemic; SARS-COV-2

\section{Introduction}

COVID-19 disease was first observed in December 2019 in Wuhan, Hubei, China and has resulted in an ongoing pandemic. It is a transmissible disease caused by severe acute respiratory syndrome coronavirus 2 , namely the SARS-CoV-2. The WHO (World Health Organization) announced this epidemic a public health emergency international distress on 31 January, 2020 and a pandemic on 11 March, 2020. Since then it has rapidly blowout through the world (see Ahn et al. (2020), Bar- On et al. (2020), Dyer (2020) for more information). As of 21 September 2020, over 31 million cases have been identified in 188 countries and territories, resulting in more than 961,000 deaths(Source: Wikipedia). Different countries have imposed different methods to control the flow in COVID-19 mortality (details see, Pike and Saini (2020), Yan et al. (2020) and others). Therefore, it is hard to compare the numbers of cases and the number of deaths among countries because of different containment and enormous differences in testing policies. This study follows the statistical tools proposed by Middelburg and Rosendaal (2020), permit a direct comparison among countries.

In this paper, the number of publicly available COVID-19 cases and the number of deaths per 100,000 from 8 March 2020 to 21 September 2020 were compared among Bangladesh, India and Pakistan. These countries are chosen for a direct comparisons in our study due to geographical, demographical, social and cultural similarities, the virus almost reached at the same time in these countries triggering a matched growth of the epidemic, almost same type of testing policies and guidelines for clinical diagnosis that probably will not be a reason differences in projected numbers of COVID-19 associated deaths.

In South Asia, after India, Bangladesh is the second most infected country, where this virus was confirmed on March 2020. By the institute of the Epidemiology, Disease Control and Research (IEDCR), the first 3 identified cases were stated on 8 March, 2020. After that this virus has blowout day by day over the entire population and the number of affected people since then has been growing. As of 21 September 2020, over 348916 cases have been identified, resulting 4939 deaths (Source: Wikipedia). At present, India has the second largest confirmed cases after USA in the biosphere and has the highest number of infected cases in Asia. The first case of this novel virus was reported on 30 January, 2020 in India, initiated. It was the number of total 100,000 cases on 19 May, 2020 and $1,000,000$ cases on $17 \mathrm{July}, 2020$. India recorded the global peak day point with 78,761 cases and exceeding the earlier top of 77,368 cases on 29 August, recorded in the US on 17 July. As of 21 September, 2020, over 5487497 cases have been identified, resulting in 87880 deaths. Pakistan presently has the sixth top number of confirmed cases in Asia, the third maximum number of definite cases in South Asia and the seventeen peak number of confirmed cases in the world. On 26 February, 2020, the virus has detected in Pakistan, where 2 cases were noted, just to mention a student who had just returned from Iran and another person in Islamabad. The daily new confirmed cases 6825 reached their peak on 15 June, 2020. Then daily cases have trended downwards. At the end of June, the number of cases become stable, showed a noteworthy decline. As of 21 September 2020, 306283 cases have been identified in Pakistan, resulting in 6420 deaths. 
The comparisons of absolute numbers of cases and deaths among countries are not that much meaningful for the following most common reasons: Sizes of target population are different, rates of per 100,000 population, the virus does not reach in all countries at the same time, all countries did not affect consistently and many others (details, see Middelburg and Rosendaal (2020), Sabillon et al. (2020)). Considering all of above very important reasons, following Middelburg and Rosendaal (2020), an unbiased direct comparison among these three countries will be established in this paper. There are many research on COVID-19 available in literature, to mention a few, Middelburg and Rosendaal (2020), Bar-On at al. (2020), Kwok et al. (2020), Ogundokun et al. (2020), Petropoulos and Makridakis (2020), Siegrist, and Kibria (2020) among others. This paper is organized as follows: Section 2 contains data and in depth graphical comparisons among three countries. Finally, some concluding remarks are outlined in section

\section{COVID-19 data and method}

This section describes comparability of data among the three countries.

\section{Comparison among three countries using a raw data}

Numbers of cases and deaths of selected countries between 8 March, 2020 to 21 September, 2020 were obtained from the European Union Open Data Portal, where data on worldwide numbers of reported cases and numbers of reported deaths for the COVID-19 pandemic are updated daily (EU(2020)). The number of cases and the number deaths per 100,000 thousands for these three countries are plotted in Figures 1 and 2 respectively with respect to day and descriptive measures are reported in Tables 1 to 2 .

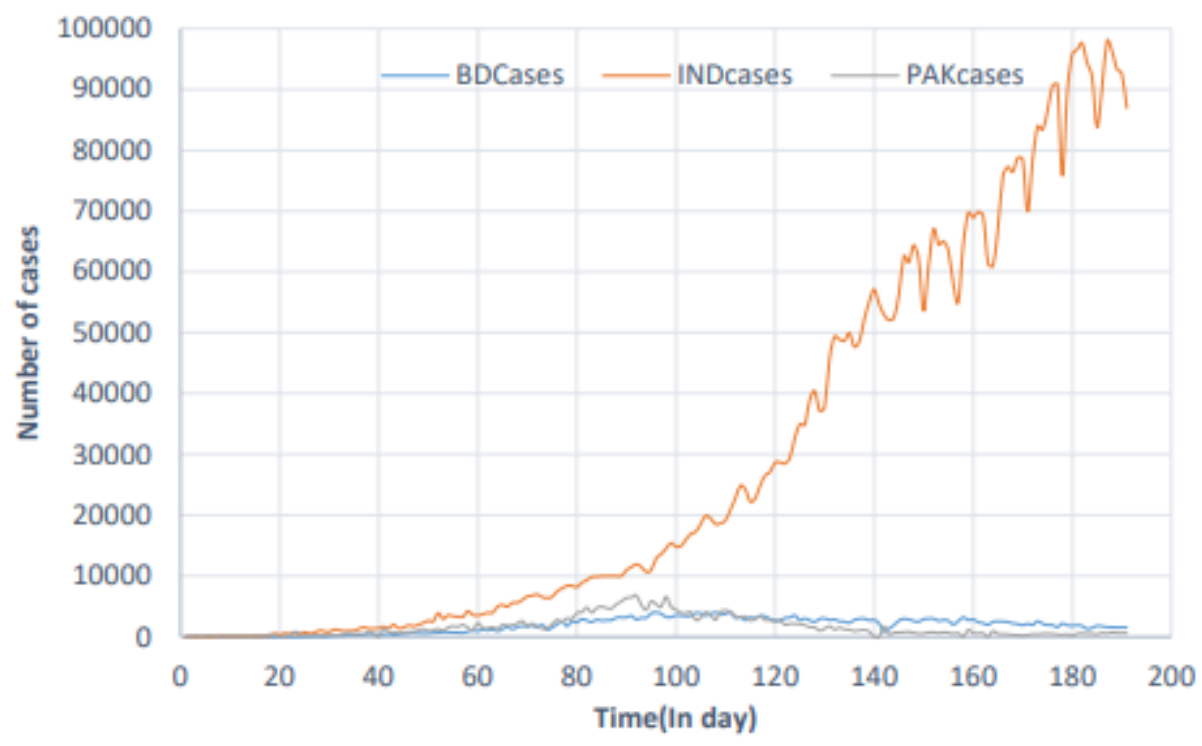

Figure 1: Cumulative number of cases in Bangladesh, India and Pakistan

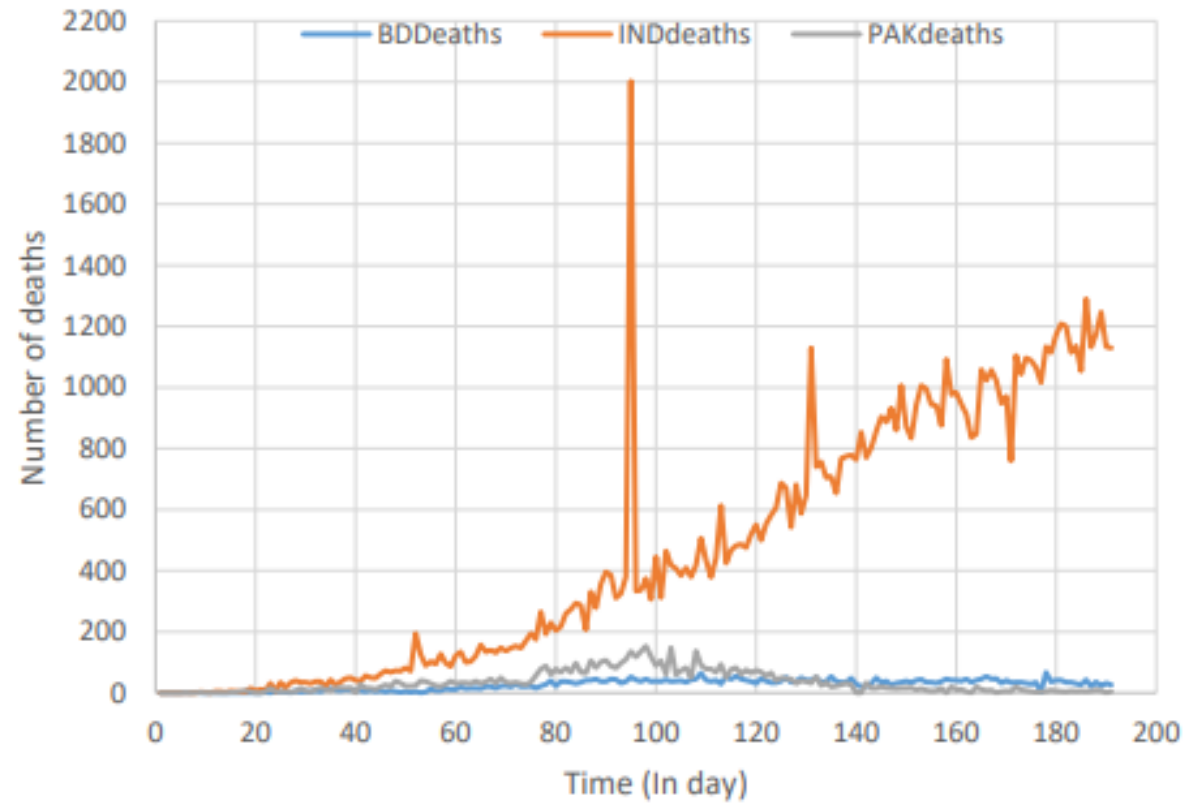

Figure 2: Cumulative number of deaths in Bangladesh, India and Pakistan 


\begin{tabular}{|l|c|c|c|c|c|}
\hline Countries & Mean & Median & SD & Skewness & Kurtosis \\
\hline Bangladesh & $1.8268 \mathrm{e}+03$ & 1950 & $1.2342 \mathrm{e}+03$ & -0.1389 & 1.7167 \\
\hline India & $2.8730 \mathrm{e}+04$ & 12881 & $3.1207 \mathrm{e}+04$ & 0.8343 & 2.2556 \\
\hline Pakistan & $1.6036 \mathrm{e}+03$ & 903 & $1.6409 \mathrm{e}+03$ & 1.3071 & 3.8449 \\
\hline
\end{tabular}

Table 1: Numerical measures of number of COVID-19 cases for all selected countries

\begin{tabular}{|l|c|c|c|c|c|}
\hline Countries & Mean & Median & SD & Skewness & Kurtosis \\
\hline Bangladesh & 25.8586 & 32 & 17.2281 & -0.1554 & 1.8089 \\
\hline India & 460.1047 & 375 & 416.3488 & 0.6379 & 2.4993 \\
\hline Pakistan & 33.6126 & 33.6126 & 35.1455 & 1.2873 & 4.0134 \\
\hline
\end{tabular}

Table 2: Numerical measures of number of COVID-19 deaths for all selected countries

In Figures 1 and 2, we have plotted the number of cases and deaths per 100,000 from March 8, 2020 to September 21, 2020 for these three countries. From Figures 1 and 2, it is observed that the numbers of infected cases and deaths in case of India are much higher as compare to Bangladesh and Pakistan. In case of Bangladesh, number of cases are gradually increasing up to 100 days then there was a declining pattern observed gradually. In case of India (see Figure 1), there was an increasing pattern observed gradually. For Pakistan, number of cases were increasing up to 100 days then there was a decreasing pattern observed as compare to Bangladesh at the end of 21 September, 2020. It is notice that as compare to India and Bangladesh, the number of deaths are much lower in Pakistan (see Figure 2).

\section{Comparison among three countries using adjusted cumulative percentage}

To enable a direct comparison among countries, for each country, cumulative numbers of cases and deaths were expressed as proportions of the cumulative numbers on the day 25 . Resulting percentages plotted against synchronized time in Figures 3 and 4 for number of cases and number of deaths respectively. It can be seen from the Figure 3, the growth of the epidemic seems dissimilar among three countries. Comparing the number of deaths as a percentage of the number on day 25 after the first death, allows a direct comparison among countries. Figure 4 shows a minor flattening of the curve for Bangladesh and Pakistan as compare to India with the most extremely developed epidemic. It also show the development of death in the India, where the epidemic developed much more rapidly as compare to Bangladesh and Pakistan. As a final point, these figures show the time-based growth of the epidemic in Pakistan, which was much steadier. Overall, findings visibly illustrates that comparing numbers of cases or deaths per 100,000 inhabitants recommend vast dissimilarities among countries. Using the number of cases as a percentage of the number of cases on the 25th day, delivers a more reliable estimate of the infected percentage of the population at threat. Using the number of deaths stated as a percentage of the number of deaths on the 25 th day after the first death, affords the best straight comparison among countries. It is notice from Figures 3 and 4 that the cumulative number of cases are overlapping between India and Pakistan, whereas the cumulative number of deaths are overlapping between Bangladesh and Pakistan. Both number of cases and number of deaths are consistently increases for India.

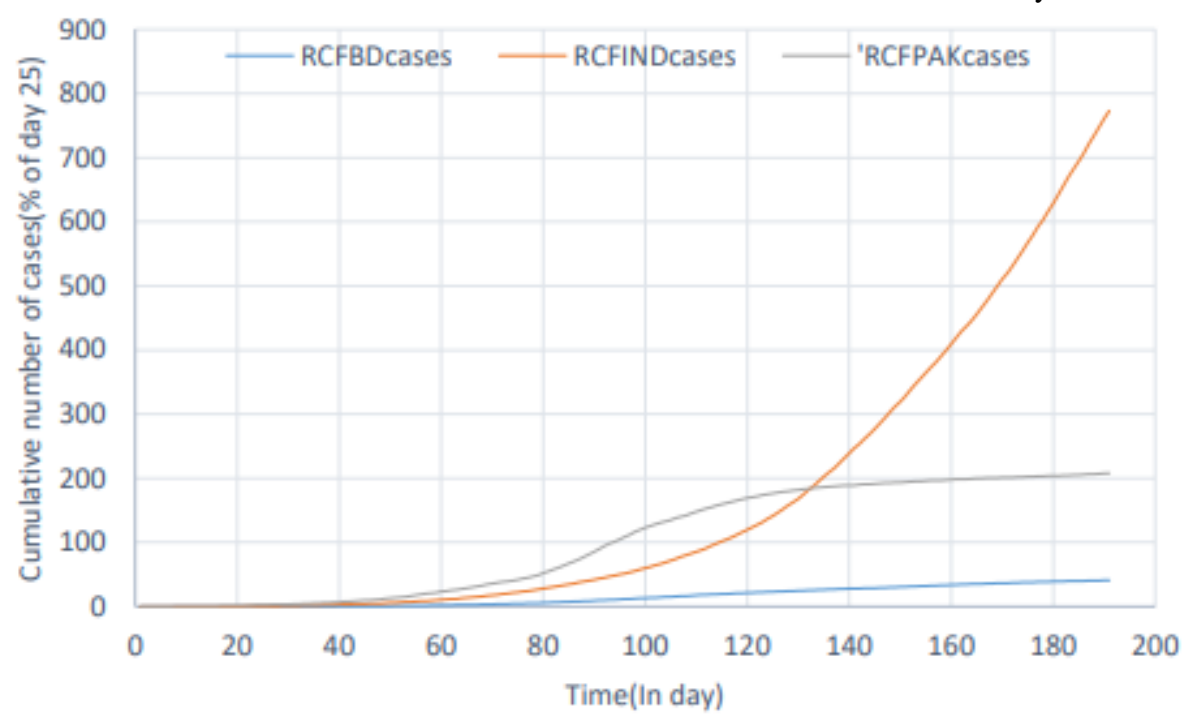

Figure 3: Cumulative number of cases as $\%$ of day 25 for Bangladesh, India and Pakistan 


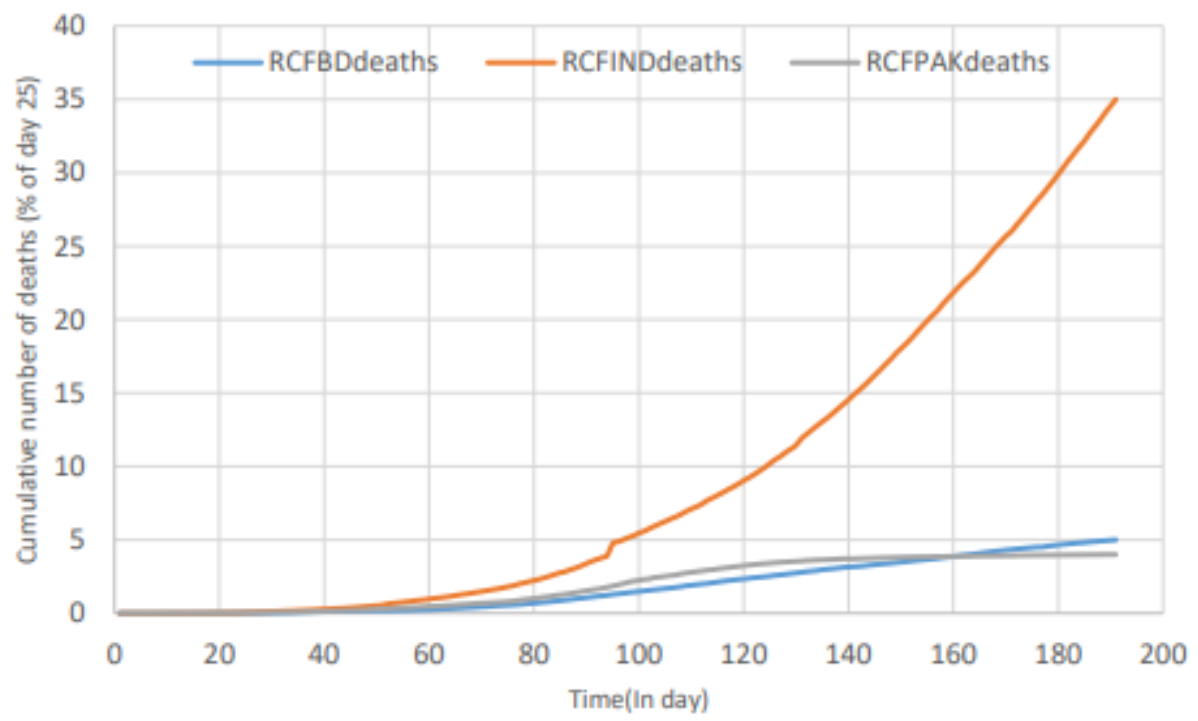

Figure 4: Cumulative number of deaths as \% of day 25 for Bangladesh, India and Pakistan

Impact of days (sensitivity analyses) using different reference days

To observe the impact of days using different reference days such as 30

plotted against synchronized time in Figures 5 to 8 respectively. Our sensitivity studies using various reference days from the reference day 25 , produced different results, which are discussed as below: and 50, percentage of the cumulative numbers of cases and deaths are

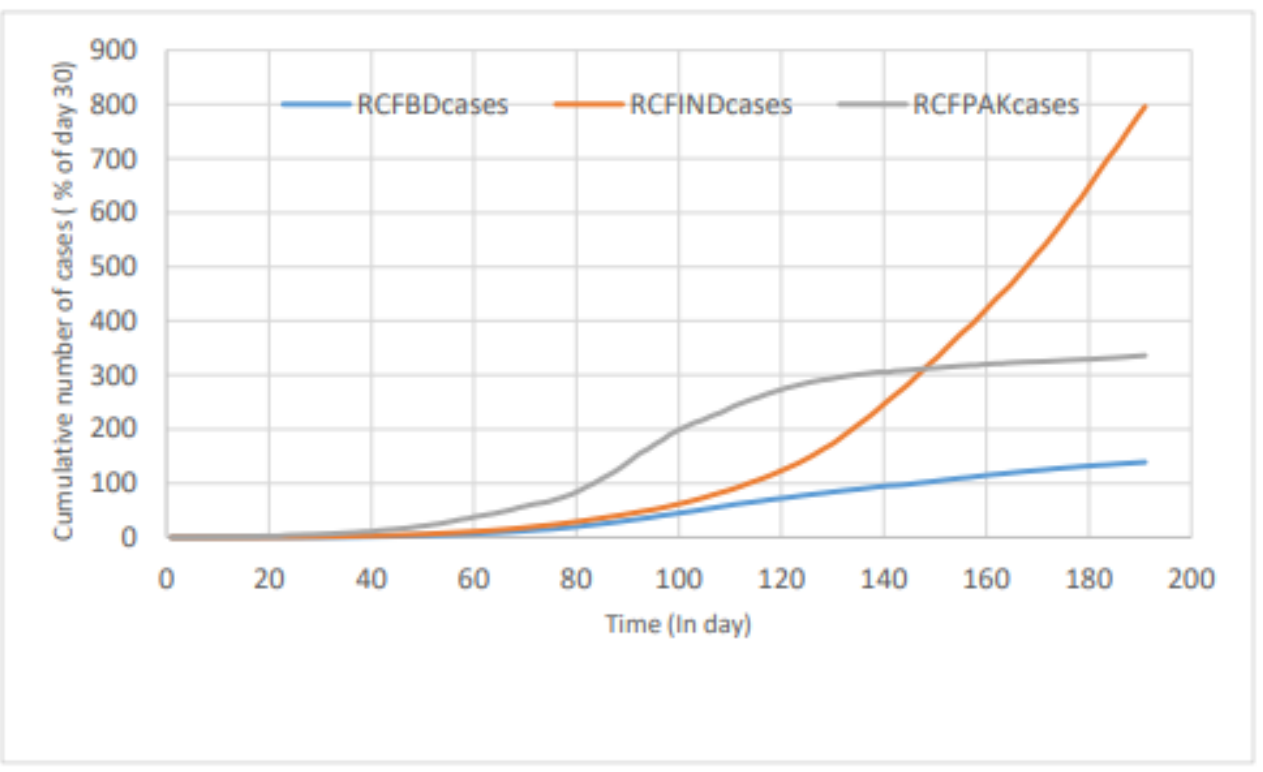

Figure 5: Cumulative number of cases as \% of day 30 for Bangladesh, India and Pakistan 


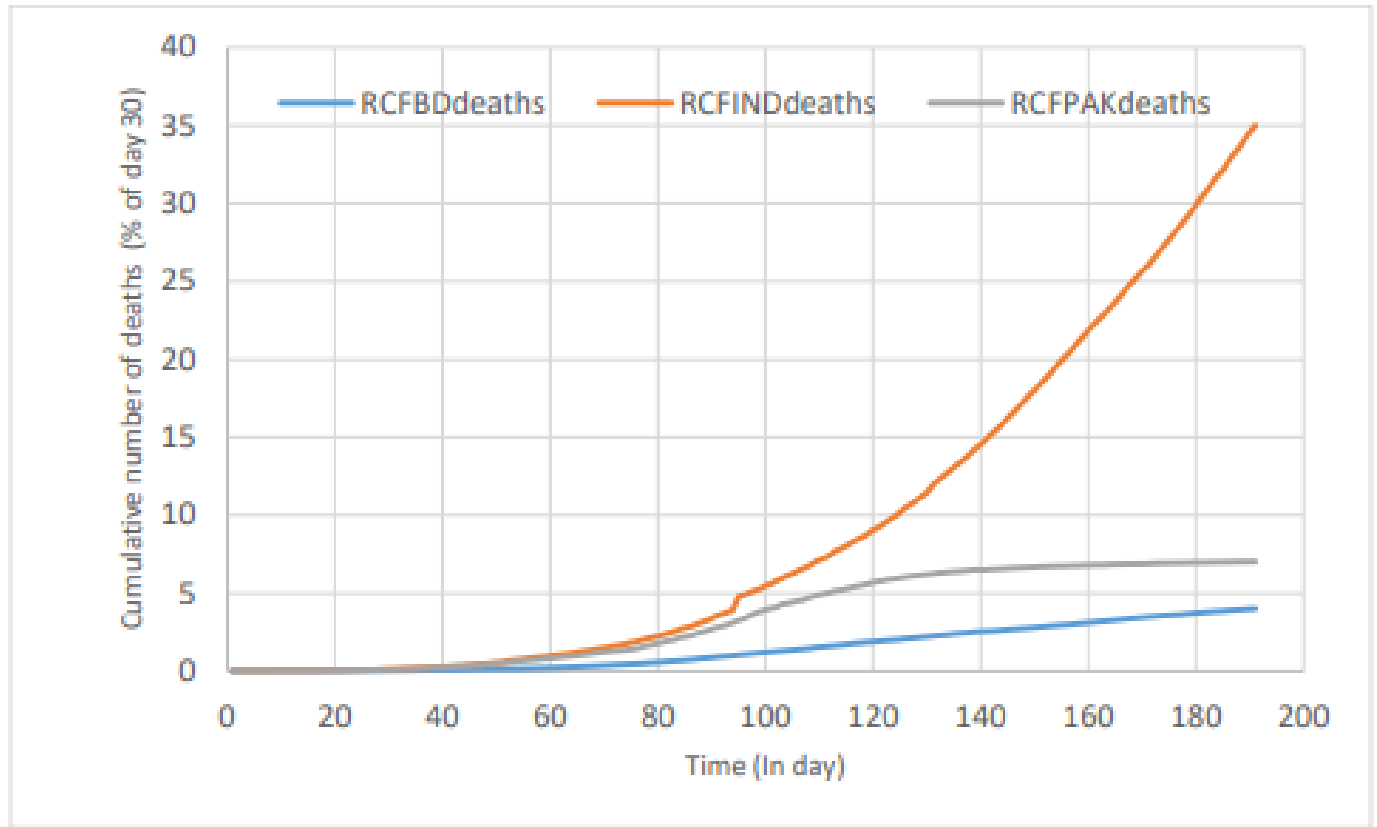

Figure 6: Cumulative number of deaths as \% of day 30 for Bangladesh, India and Pakistan

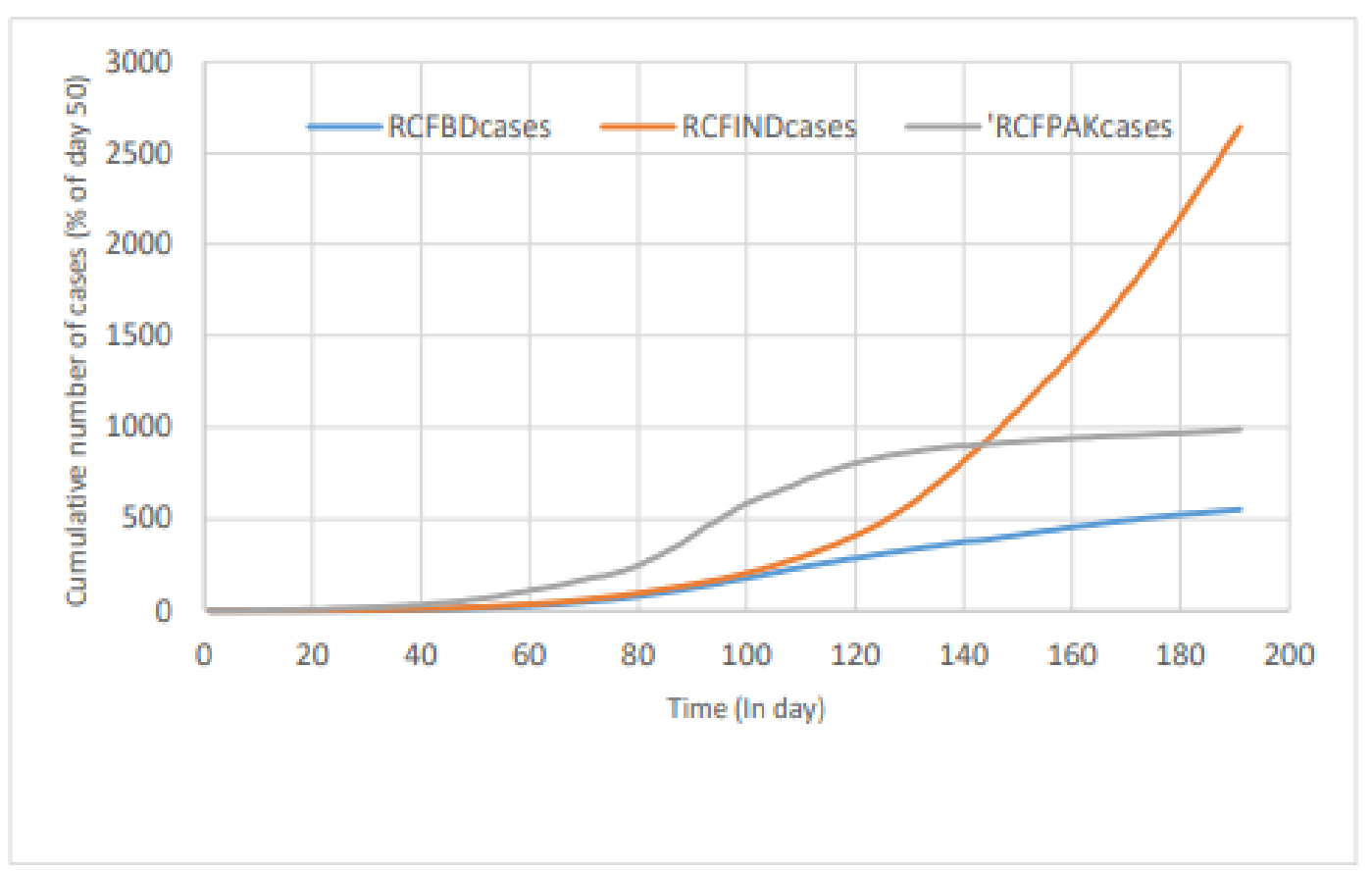

Figure 7: Cumulative number of cases as \% of day 50 for Bangladesh, India and Pakistan 


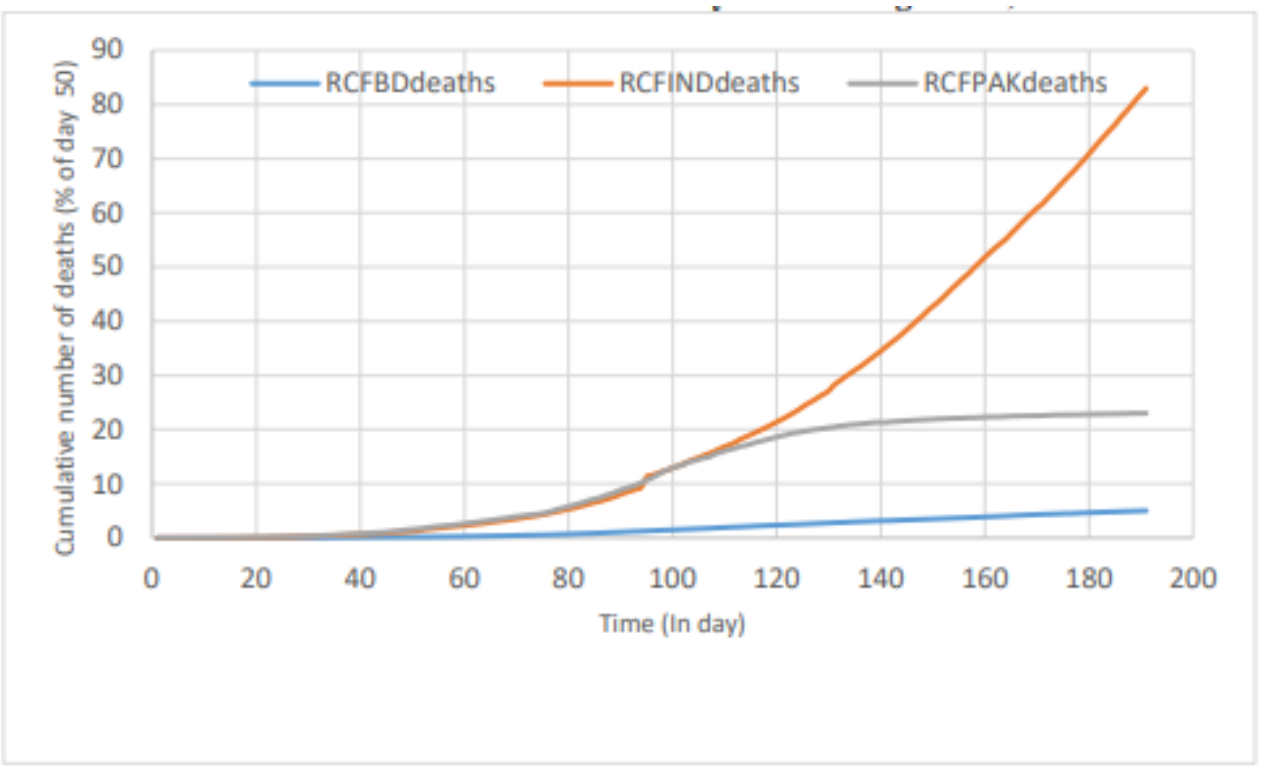

Figure 8: Cumulative number of deaths as \% of day 50 for Bangladesh, India and Pakistan

Figures 5 and 6 present percentage of the cumulative numbers of cases and deaths on the reference day 30 . Using reference day 50 , percentage of cumulative number of cases and deaths are visualized in Figures 7 and 8 respectivley. It can be seen from these figures that the growth of the epidemic seems still dissimilar among these countries. Comparing with Figures 3 and 4 (earlier reference day 25), no of cases and deaths for each countries have been increasing. It is noticeable that when using a subsequent reference day that means 30 and 50 instead of 25 , dissimilarities among countries are observed more prominent.

\section{Concluding remarks}

This paper compares the number of cases and number of deaths among Bangladesh, India and Pakistan for the period of March 8, 2020 to September 21, 2020. Comparison based on the absolute numbers are not comparable due to different factors (such as population sizes, rates of per $100,000)$ because not all countries are affected equally, vulnerability to deaths can vary among populations and a death is only reported as a COVID-19 related death if the patient was identified with the SARS-CoV2 infection. With the existing approaches and all above factors were accounted, an equitable straight assessment among countries were established. It is observed that the most extremely developed epidemic in India which developed much more quickly. It is also observed that Bangladesh had the most extreme flattening of the curve followed by Pakistan and India. Using different reference days, sensitivity analyses have been done that show that huge differences exist between number of cases and deaths among these three countries.

\section{Acknowledgements}

The authors are dedicating this paper to those who have sacrificed their lives during the Bangladesh Liberation War in 1971. They are also thankful to the referees for their comments and suggestions which certainly improved the presentation of this paper.

\section{References}

1. Ahn, D.G., Shin, H.J., M.H., Lee, S., Kim, H.S. and Myoung, J. (2020) Current status of epidemiology, diagnosis, therapeutics and vaccines for novel coronavirus disease 2019 (COVID-19). J Microbiol Biotechnol, 30, 313-324.

2. Bar-On, Y.M., Flamholz, A., Philips, R. and Milo, R. (2020) SARS-CoV-2 (COVID-19) by the numbers. Elife.

3. Dyer, O. (2020) Covid-19: hospitals brace for disaster as US surpasses China in number of cases. March News, BMJ.

4. EU Open Data Portal: COVID-19 cases worldwide. 2020.

5. Kwok, K.O., Lai, F., Wei, W,I., Wong, S.Y.S., Tang, J. (2020) Herd immunity estimating the level required to halt the COVID-19 epidemics in affected countries. J Infect.

6. Middelburg, R.A. and Rosendaal, F.R. (2020) COVID-19: How to make between-country comparison, International Journal of Infectious Diseases, 96, 477-481.

7. Ogundokun, R.O., Lukman, A.F., Kibria, B.M.G., Awotunde, J.B. and Aladetian, B.B. (2020) Predictive modeling of COVID-10 confirmed cases in Nigeria. Infectious Disease Modelling, 5, 543548.

8. Petropoulos, F. and Makridakis, S. (2020) Forecasting the novel coronavirus COVID-19. PLoS One, 15, 231-236.

9. Pike, T.W. and Saini, V. (2020) An international comparison of the second derivative of COVID-19 deaths after implementation of social distancing measures.

10. Sabillon, C.M., Guzman, C.I. and Kibria, B.M.G. (2020) Comparing COVID-19 among some highly affected states in the United States of America. Journal of Biostatistics and Epidemiology, 6(3), 179-189.

11. Siegrist, M. and Kibria, B.M.G. (2020) Predicting Total Number of Deaths using COVID-19 World Data: Application of Linear Regression Model. To appear International Journal of Industrial and Operations Research. 3:008.

12. Yan, Y., Shin, W.I., Pang, Y.X., Meng, Y., Lai, J, and You, C. (2020) The first 75 days of novel coronavirus (SARS-CoV-2) outbreak: Recent advances, prevention and treatment, Int J Environ Res Public Health, 17, 2323. 